\title{
VISIBILITÉ DES PUBLICATIONS DES GÉOGRAPHES DE L'ULIÈGE DANS LES BASES DE DONNÉES BIBLIOGRAPHIQUES COMMERCIALES ET GOOGLE SCHOLAR
}

\author{
Simona STIRBU, Serge SCHMITZ, Ninfa GRECO
}

\begin{abstract}
Résumé
Cet article s'intéresse à la visibilité de la littérature scientifique produite par les géographes de l'ULiège, à travers des bases de données bibliographiques traditionnelles et du moteur de recherche Google Scholar. La contribution du répertoire institutionnel ORBi dans la meilleure visibilité des références est évaluée également.

Concrètement, une analyse du référencement d'un échantillon de publications par les bases de données commerciales multidisciplinaires, Scopus et WoS, et spécialisée GeoRef, ainsi que par le moteur de recherche GS est effectuée. D'autres facteurs tels que la langue de publication et la distribution entre ST et SHS ont été pris en compte.

Les résultats montrent que la distribution des publications des membres du département de Géographie entre les types de documents est différente en fonction de l'orientation du sujet vers les SHS ou les ST, même si les articles de périodiques constituent le principal type de publications, quelle que soit la sous-discipline. En SHS, les chapitres et parties de livres ainsi que les articles et communications sont majoritairement en français. Les performances de GS sont supérieures à celles des BD pour notre échantillon de publications des géographes de l'ULiège.

En conclusion, le répertoire institutionnel ORBi contribue incontestablement à améliorer les performances de GS quant au recouvrement de notre échantillon de publications et à donner une meilleure visibilité de la littérature francophone en Géographie.
\end{abstract}

\author{
Mots-clés \\ littérature en géographie, analyses des bases de données bibliographiques, Google Scholar, WoS, \\ GeoRef, ULiège
}

\begin{abstract}
This article firstly examines the visibility of a sample of scientific literature produced by ULiège geographers in the commercial bibliographic databases and the Google Scholar search engine. The role of our institutional repository, ORBi, in the better visibility of references is also assessed. In our study we analysed the referencing of a sample of publications by the multidisciplinary commercial databases, Scopus and WoS, the specialized GeoRef, and the search engine GS. Factors such as the language of publication, the distribution between $S \& T$ and $S S \& H$ were also considered in the study.

The results show that the distribution of the publications of the members of the Geography Department between the types of documents is different according to the orientation of the subject towards $S S \& H$ or $S \& T$, even if the journal articles constitute the main type of publications, whatever the sub-discipline. In SS\&H, the chapters, the parts of books, as well as the articles and communications are mainly in French.

Compared to commercial DBs the performances of GS are superior for our sample of publications. These findings support the contribution of the ORBi institutional repository to improving GS's 'performance with regard to the indexing of geographers' publications and to giving better visibility of French-speaking geographic literature.
\end{abstract}

Keywords

geographical literature, bibliographic database analysis, Google Scholar, WoS, GeoRef, ULiège

\section{INTRODUCTION ET REVUE DE LA LIT- TÉRATURE}

Durant les dernières décennies, le développement du support numérique a révolutionné le paysage documentaire de la communauté scientifique. L'édition en format électronique des périodiques scientifiques s'est généralisée dans un premier temps, puis celle d'autres types de documents. Des consortiums se sont mis en place ouvrant l'accès à des collections complètes de différents éditeurs commerciaux, et le mouvement OAI (Open Access Initiative, 1999) a contribué à mettre à la portée de tous une masse considérable d'informations.

En parallèle, de nouveaux outils de recherche documentaire sont apparus sur le web : des bases de données bibliographiques commerciales comme 
Scopus en 2004, des moteurs de recherche d'usage gratuit comme Google Scholar (GS) en 2004 également ou encore des « réseaux sociaux » destinés aux scientifiques comme ResearchGate en 2008.

Ce phénomène n'a pas manqué de susciter la réflexion des professionnels de l'information et des chercheurs et s'est traduit par la production d'une littérature abondante sur le sujet. Néanmoins, les études se focalisant sur une discipline scientifique déterminée comme la Géographie sont relativement plus rares.

Sur ce constat, nous avons précédemment comparé l'utilité de Google Scholar par rapport à trois bases de données traditionnelles (GeoRef, Web of Science et Francis) pour la recherche documentaire en Géographie (Stirbu et al., 2015). Nous avons montré que les résultats d'une recherche par mots-clés dans Google Scholar couvraient largement les résultats d'une même recherche effectuée dans les bases de données commerciales. De plus, Google Scholar apportait son lot de références propres, en particulier en Géographie humaine (articles de périodiques évidemment mais aussi d'autres types de documents comme des rapports, thèses, comptes-rendus de conférences et livres ou chapitres de livres).

Dans le présent travail ${ }^{1}$, nous voulons vérifier et comparer la visibilité de la littérature scientifique produite par les géographes de ULiège dans GS par rapport aux bases de données commerciales.

Comme ULiège dispose d'un répertoire reconnu au niveau mondial, ORBi, et d'une politique institutionnelle forte en matière de dépôt des publications (Thirion, 2016), nous nous sommes interrogés sur l'apport de ce répertoire institutionnel combiné au moissonnage de son contenu par GS dans la visibilité des publications des géographes de l'Université de Liège. À notre connaissance, ceci est la première étude de visibilité des publications en sciences géographiques intégrant la notion de dépôt dans un répertoire institutionnel.

D'une façon plus générale, cette étude s'inscrit dans la lignée des études comparant les performances des outils de recherche bibliographique et portant sur l'apport de l'Open Access.

Peu après la mise en production de Scopus et GS en 2004, une des plus vastes analyses comparatives a été effectuée par Bosman et al., (2006) notamment pour Scopus, WoS et GS. Leurs résultats ont montré que Scopus avait une couverture des disciplines des Sciences de la terre ainsi que de la Géographie sociale et de l'Aménagement du territoire $10 \%$ plus importante que celle de WoS.

Les auteurs ont aussi précisé que seulement $13 \%$ des publications indexées par GeoRef étaient couvertes par Scopus, GeoRef renseignant une plus grande diversité de types de documents. En revanche, Scopus de par son contenu multidisciplinaire permet la recherche dans plusieurs disciplines, qui sont parfois connexes. Enfin, les auteurs suggèrent que les meilleures performances de Scopus par rapport à WoS pourraient être dues à l'apport de Geobase, intégralement incluse dans Scopus.

Quant à GS, toujours dans la même étude, il se différencie plus nettement de Scopus et de WoS par la couverture de citations que les deux bases de données entre elles. Ceci s'explique par la représentation d'autres langues que l'anglais et de différents types de publications, beaucoup plus encore pour la Géographie humaine que pour les Sciences de la terre.

Ensuite, une série d'études comparatives, sans lien spécifique avec la Géographie, ont suivi. Meho et Yang (2007) ont comparé WoS, Scopus et GS dans le domaine des Sciences de l'information. Les auteurs ont également constaté que par rapport à WoS et Scopus, GS se distinguait dans la couverture des actes de conférences ainsi que des périodiques internationaux publiés dans d'autres langues que l'anglais. Scopus et GS seraient des outils complémentaires à WoS pour la recherche documentaire. Pour GS, le temps consacré au traitement des données est cependant beaucoup plus important.

Vieira et Gomes (2009) ont travaillé sur un échantillon d'articles correspondant à la production scientifique de 16 universités portugaises entre 2000 et 2007. Ils ont compté les documents référencés dans WoS et Scopus. Leur conclusion générale indique qu'environ $2 / 3$ des documents se trouvent dans les deux bases de données alors que $1 / 3$ sont uniquement répertoriés par l'une des deux.

De Sutter et Van Den Oord (2012) ont utilisé WoS, Scopus, $\mathrm{ACM}^{2}$, GS et CiteSeer ${ }^{3}$ pour répertorier les publications de trois informaticiens belges. Ils ont 
constaté que les couvertures de WoS, Scopus, et de GS sont excellentes pour les articles de périodiques. WoS et Scopus indexent une sélection de conférences et de périodiques à fort impact. Globalement pour les Sciences informatiques, la couverture de WoS est comparable à celle de Scopus. GS couvre plus de « proceedings » et de conférences que les autres bases de données.

Adriaanse et Rensleigh (2013) ont comparé WoS, Scopus et GS afin d'identifier la ressource bibliographique la plus représentative pour les publications en sciences environnementales en Afrique du Sud. Leurs résultats indiquent une meilleure performance de WoS en matière de couverture et d'apport de références « uniques ». Les auteurs ont également relevé que GS présentait plus d'incohérences et d'erreurs quant à l'orthographe des noms d'auteurs et les volumes et numéros de publication.

Bartol et al., (2014) ont analysé le recensement par Scopus et WoS des 50000 documents de recherche publiés en Slovénie entre 1996 et 2011. Ils ont constaté que Scopus indexe plus de documents que WoS dans tous les domaines de recherche. Les différences sont particulièrement évidentes en SHS ainsi qu'en Ingénierie et techniques.

Cavacini (2015) a recherché un échantillon d'articles provenant d'un répertoire institutionnel italien dans INSPEC ${ }^{4}$, Scopus, WoS et DBLP ${ }^{5}$. Ses résultats montrent une meilleure performance de Scopus pour retrouver les publications en Informatique, par rapport à WoS. Scopus et DBLP indexent le plus grand nombre d'articles « uniques », mais chacune des bases de données indexe sa part de références « uniques » et elles sont donc complémentaires.

Dans une autre étude récente, réalisée par Gorraiz et al., (2016), on retrouve des données portant sur la Géographie. Cette étude a été réalisée dans le but d'évaluer 40 candidatures à des postes du Département de Géographie de l'Université de Vienne, dans des sous-disciplines variées comme la Géographie de la population et la démographie, la Géographie économique, la Géo-écologie et la Géographie économique et sociale. Il s'agissait de mettre en évidence les différences éventuelles dans les pratiques de publication. Pour la Géographie de la population et la démographie, les publications des candidats ainsi que les citations reçues ont été recherchées en interrogeant en parallèle trois outils bibliographiques : WoS, Scopus et GS. Pour la Géographie économique, WoS et GS ont été retenus tandis que pour la Géo-écologie et la Géographie économique et sociale, seul WoS a été utilisé.

Notons par ailleurs que les auteurs ont détecté des différences dans les pratiques de publication des géographes relevant de la Géographie physique d'une part et de la Géographie humaine d'autre part. Les chercheurs travaillant dans les sous-disciplines orientées vers les Sciences naturelles ont tendance à publier principalement des articles, dans des périodiques en anglais et à haut facteur d'impact, qui sortent de leur discipline principale. Les chercheurs travaillant dans des sous-disciplines orientées vers les SHS, quant à eux, publient dans des périodiques plutôt orientés vers la Géographie et majoritairement des parties et chapitres de livres. Les auteurs n'ont pas constaté de différence majeure en utilisant WoS ou Scopus, et ils encouragent l'usage de GS en tant qu'outil complémentaire, notamment pour la recherche des livres et de la littérature grise, car ce dernier offre une meilleure couverture.

La revue de la littérature montre que les études s'accordent généralement sur le fait que les performances des bases de données commerciales sont similaires et que GS, quand il est pris en compte, fournit des bons résultats et complète les bases commerciales.

Globalement, il semble que GS ait amélioré sa couverture au fil des ans, en particulier dans le domaine des SHS (Jamali et Nabavi, 2015), ce qui pourrait distinguer le moteur de recherche des deux bases de données multidisciplinaires évoquées précédemment.

Un autre atout considérable de GS est le lien direct vers le texte intégral qu'il fournit (lorsqu'il est disponible), que ce soit via les répertoires institutionnels, ResearchGate, ou directement sur les sites des éditeurs (Mikki et al., 2018).

Avec le développement de l'Open Access (OA) et l'encodage massif des articles dans les répertoires institutionnels, il serait logique de penser que les articles déposés dans des répertoires en $\mathrm{OA}$, donc susceptibles d'être plus lus et plus souvent cités, aient un impact plus important (Norris, 2008). Cette hypothèse a fait l'objet de différentes études (Davis et Fromerth, 2007 ; Davis et Price, 2006 ; 
Davis et Walters, 2011 ; Davis, 2010 ; Gorraiz et al., 2014 ; Guerrero-Bote et Moya-Anegón, 2014 ; Harnad et al., 2004 ; Moed, 2007). Les dernières études (Archambault et al., 2016; Jamali et Nabavi, 2015 ; Mikki et al., 2018 ; Piwowar et al., 2018) font état d'un impact de citation jusqu'à $50 \%$ plus important pour ces documents.

\section{OBJECTIFS ET HYPOTHÈSES}

Le premier objectif de notre travail est d'examiner et de qualifier un échantillon de publications des géographes de ULiège, sélectionné à partir d'ORBi, pour une mise en perspective avec la littérature.

Le second objectif est de déterminer dans quelle mesure la littérature scientifique produite par les géographes de ULiège est répertoriée par les bases de données commerciales WoS, Scopus, GeoRef et par GS.

En intégrant un nouvel élément, soit le dépôt des références dans ORBi par les membres du Département de Géographie, nous pourrons déterminer la contribution du répertoire institutionnel à la visibilité de cette littérature.

Sur base de la revue de la littérature, nous posons les hypothèses suivantes :

Hypothèse 1 : Selon que l'on considère l'orientation vers les SHS ou les ST, la répartition des publications de notre échantillon entre les chapitres ou parties de livres et les articles de périodiques ou communications est nettement différente ;

Hypothèse 2: Quelle que soit la sous-discipline, l'article de périodique est la principale voie de publication;

Hypothèse 3 : Quelle que soit la sous-discipline ou le type de document, l'anglais est la principale langue de publication;

Hypothèse 4 : Grâce au moissonnage d'ORBi, GS retrouve toutes les publications des géographes de ULiège, déposées dans le répertoire institutionnel ; Hypothèse 5 : Les références retrouvées uniquement dans GS, exclusivement grâce au moissonnage d'ORBi, sont nombreuses, surtout pour la Géographie humaine ;

Hypothèse 6: Si on fait abstraction des références en provenance d'ORBi, une proportion plus élevée de références est retrouvée par GS en comparaison des bases de données bibliographiques commerciales ;
Hypothèse 7 : Le recouvrement de WoS et Scopus est très important et seul Scopus se distingue par un apport significatif de références supplémentaires par rapport à WoS, relevant souvent des SSH ;

Hypothèse 8 : Dans l'absolu, GeoRef couvre moins de références que WoS et Scopus mais se distingue par un apport de références supplémentaires en Géographie physique par rapport aux deux autres bases de données commerciales ;

Hypothèse 9 : Du fait de leur présence dans le répertoire institutionnel $\mathrm{ORBi}$, les publications en langue française des géographes liégeois publiées dans les périodiques « locaux » sont plus visibles via GS que via les bases de données commerciales.

\section{MÉTHODES}

Un rapport reprenant les références des livres, parties et chapitres de livres ainsi que articles de périodiques et communications, publiés entre 2000 et 2014 par les membres du Département de Géographie (ULiège) et déposés dans ORBi, a été généré en septembre 2015.

Les métadonnées (auteurs, titres, années de publication, type de publication, périodique, ISSN) de ces références ont été téléchargées et enregistrées dans des fichiers Excel. Les erreurs d'encodage ont été repérées et corrigées.

Une référence pouvant être associée à plusieurs disciplines dans ORBi, pour le descriptif nous avons pris en compte la première discipline indiquée par le déposant, en supposant qu'il s'agit de la discipline principale de la référence.

Afin de statuer sur la couverture des références par les outils bibliographiques, chaque référence de l'échantillon a été recherchée dans GS, GeoRef et Scopus.

Notre démarche a été limitée pour les recherches dans WoS, car l'Université de Liège n'a plus accès à cette base depuis 2011. Nous avons adapté notre méthode et nous nous sommes appuyés sur les informations d'un fichier Excel que nous avons pu obtenir par le service « clients » pour une recherche par titres de périodiques. Les recherches pour les parties et chapitres de livres n'ont pas été effectuées dans WoS.

Les listes de références de documents couverts par les différents outils ont été établies et comparées. 
Les références « uniques » de GS et celles provenant exclusivement d'ORBi ont également fait l'objet d'un relevé spécifique.

Les pourcentages des articles et communications couverts par un ou plusieurs outils bibliographiques ont été calculés. Afin d'estimer l'apport d'ORBi, deux types de pourcentages ont été calculés pour GS : ceux qui prenaient en compte les résultats provenant du moissonnage d'ORBi et ceux qui les écartaient. Les recouvrements des outils entre eux ont été représentés avec les diagrammes de Venn.

\section{RÉSULTATS}

L'échantillon de publications comprend 146 chapitres ou parties de livres ainsi que 405 articles et 48 communications publiés dans des périodiques. La répartition des chapitres et parties de livres par discipline (ORBi), ainsi que des articles et communications, est reprise dans le Tableau 1.

\section{A. Les chapitres et parties de livres}

Les chapitres et parties de livres représentent $24 \%$ de l'échantillon total. Ce pourcentage est relativement équivalent à celui indiqué par Gorraiz et al., (2016), selon lequel un tiers des publications du Département de Géographie et de recherches régionales de l'Université de Vienne étaient des chapitres de livres tandis qu'un dixième étaient des livres.
Les « Sciences sociales \& comportementales, psychologie » comptent le plus grand nombre des publications sous forme de chapitres ou de parties de livres (87), dont la majorité (61) traitent plus précisément de « Géographie humaine \& démographie $»$.

Vient ensuite la « Physique, chimie, mathématiques $\&$ sciences de la terre » qui compte 50 références de chapitres et parties de livres, dont 46 en « Sciences de la terre $\&$ géographie physique $»$.

Toutes disciplines confondues, les SHS comptent 91 publications sous forme de chapitres ou parties de livres, soit $43 \%$ du total des publications en SHS (210 publications) tandis que les ST comptent 55 publications sous forme de chapitres ou parties de livres, soit $14 \%$ du total des publications en ST (389 publications).

Il apparaît donc une nette différence dans la balance entre les chapitres ou parties de livres et les articles de périodiques ou communications, selon que l'on considère les SHS ou les ST.

Pour ce qui concerne la langue de publication, toutes disciplines confondues, une majorité de $57 \%$ (83) de chapitres ou parties de livres sont publiés en français tandis que $42 \%$ (61) sont publiés en anglais. Pour les SHS, le pourcentage de publications en français monte à $74 \%$ (67) pour $24 \%$ (22) en anglais. En ST, le pourcentage de publications

\begin{tabular}{|l|c|c|c|c|c|c|}
\hline Discipline & $\begin{array}{c}\text { Nb. } \\
\text { Ch. } \\
\text { Livres }\end{array}$ & Ang. & Fr. & $\begin{array}{c}\text { Nb. } \\
\text { Art. }\end{array}$ & Ang. & Fr. \\
\hline Arts \& sciences humaines & 1 & 1 & & 3 & 2 & 1 \\
\hline Droit, criminologie \& sciences politiques & 3 & 1 & 2 & 1 & & 1 \\
\hline Ingénierie, informatique \& technologies & & & & 28 & 19 & 9 \\
\hline Physique, chimie, mathématiques \& sciences de la terre & 50 & 39 & 11 & $267^{* *}$ & 197 & 68 \\
\hline Sciences sociales \& comportementales, psychologie & $87 *$ & 20 & 65 & $82^{* * *}$ & 11 & 70 \\
\hline $\begin{array}{l}\text { Sciences du vivant (Sciences de l'environnement \& } \\
\text { écologie) }\end{array}$ & 5 & & 5 & 36 & 21 & 15 \\
\hline Sciences économiques \& de gestion & & & & 33 & 3 & 30 \\
\hline Sciences de la santé humaine & & & & 3 & 3 & \\
\hline $\begin{array}{l}* 1 \text { référence en espagnol et 1 référence en allemand } \\
* * 2 \text { références en néerlandais } \\
* * * 1 \text { référence en portugais }\end{array}$ & & & & & \\
\hline
\end{tabular}

Tableau 1. Disciplines et langues des différents types de documents relevés dans ORBi, les intitulés en gras sont les disciplines reprises dans la catégorie ST 
en français descend à $29 \%$ (16) et le pourcentage de publications en anglais monte à $71 \%$ (39). Les autres langues sont utilisées de manière tout-à-fait marginale, en SHS.

La différence entre SHS et ST se confirme également pour ce qui est de la langue de publication.

\section{B. Recensement des chapitres ou parties de livres}

Pour ce qui est du recensement des chapitres ou parties de livres par les différents outils bibliographiques, GS référence $100 \%$ des publications (Tableau 2). Si on faisait abstraction des 96 références retrouvées uniquement grâce à un lien vers ORBi, les performances du moteur de recherche diminueraient considérablement avec seulement 50 chapitres ou parties de livres référencés $(33,5 \%)$.

Des 50 chapitres ou parties de livres retrouvés par GS sans l'apport d'ORBi, 17 sont en " Sciences sociales \& comportementales, psychologie », dont 16 traitent plus précisément de " Géographie humaine \& démographie ». 31 chapitres et parties de livres sont en «Physique, chimie, mathématiques $\&$ sciences de la terre », dont 28 en « Sciences de la terre $\&$ géographie physique $»$.

Des 96 chapitres ou parties de livres retrouvés grâce à $\mathrm{ORBi}, 74$ sont en « Sciences sociales \& comportementales, psychologie », dont la majorité (50) traitent plus précisément de « Géographie humaine \& démographie ». 19 chapitres et parties de livres sont en «Physique, chimie, mathématiques $\&$ sciences de la terre », dont 18 en « Sciences de la terre \& géographie physique ».

Scopus ne retrouve que 6 références, toutes en «Physique, chimie, mathématiques \& sciences de la terre » et dont 5 plus spécifiquement en « Sciences de la terre \& géographie physique ».

Quant à GeoRef, 5 références sont retrouvées par cette base de données disciplinaire, toutes en Sciences de la terre \& géographie physique.

\section{Les articles de périodiques et les communi- cations}

Les articles de périodiques et les communications représentent $76 \%$ (453) de l'échantillon total.
Par rapport aux chapitres et parties de livres, la répartition du nombre de publications entre les deux disciplines principales s'inverse (Tableau 1) avec une majorité de 267 publications en " Physique, chimie, mathématiques \& sciences de la terre ", dont 247 publications en « Sciences de la terre \& géographie physique », et avec 82 publications en «Sciences sociales \& comportementales, psychologie » dont 49 publications en « Géographie humaine \& démographie ». La sous-discipline «Sciences de la terre \& géographie physique » compte le plus grand nombre de références.

Toutes disciplines confondues, les ST comptent 334 publications sous forme d'articles de périodiques ou de communications, soit $86 \%$ du total des publications en ST (389 publications) tandis que les SHS comptent 119 publications sous forme d'articles de périodiques ou de communications, soit $57 \%$ du total des publications en SHS (210 publications).

Pour ce qui concerne la langue de publication, toutes disciplines confondues, la tendance relevée pour les chapitres et parties de livres s'inverse ici, avec une majorité de $57 \%$ (256) de publications rédigées en anglais et avec $43 \%$ (194) des publications en français. Les autres langues sont toujours utilisées de manière marginale.

Pour les ST, le pourcentage de publications en anglais monte à $72 \%$ (240) pour $28 \%(92)$ en français. En SHS, le pourcentage de publications en anglais descend à $13 \%$ (16) et le pourcentage de publications en français monte à $86 \%$ (102).

Cette fois encore, la différence entre SHS et ST se confirme pour ce qui est de la langue de publication.

\section{Recensement des articles et des communications}

GS retrouve $100 \%$ des 453 articles et communications de notre échantillon (Tableau 3). À l'analyse, il s'avère que 68 de ces références sont retrouvées par GS exclusivement par les liens établis avec ORBi et qu'elles sont toutes « uniques » au moteur de recherche.

Même en faisant abstraction des références issues exclusivement d'ORBi, GS présente la meilleure performance avec 385 des références couvertes (84,9\%), dont 252 des 267 (94\%) de « Physique, chimie, mathématiques \& sciences de la terre » et 
les bases de données bibliographiques commerciales et Google Scholar

\begin{tabular}{|l|c|c|c|c|}
\hline Databases & GS & GS (-) ORBi & Scopus & GeoRef \\
\hline Nb. ref. & 146 & 50 & 6 & 5 \\
\hline$(\%)$ & 100 & 33,5 & 4,1 & 3,4 \\
\hline
\end{tabular}

Tableau 2. Nombres et pourcentages de chapitres ou parties de livres retrouvés par les outils bibliographiques

\begin{tabular}{|l|c|c|c|c|c|}
\hline Databases & GS & GS (-) ORBi & Scopus & WoS & GeoRef \\
\hline Nb. ref. & 453 & 385 & 224 & 167 & 150 \\
\hline$(\%)$ & 100 & 84,9 & 49,4 & 36,8 & 32,8 \\
\hline
\end{tabular}

Tableau 3. Nombres et pourcentages des articles retrouvés dans les outils bibliographiques

55 des $82(67 \%)$ de « Sciences sociales \& comportementales, psychologie ». À titre de comparaison, Gorraiz et al., (2016) ont retrouvé dans GS 74,6 \% d'articles publiés en Démographie et Géographie de la Population et $91 \%$ en Géographie Economique.

Le moteur de recherche GS est suivi par Scopus avec 49,4\% (224) de références couvertes (Tableau 3), dont 162 des 224 (72,3 \%) en « Physique, chimie, mathématiques \& sciences de la terre » et 14 des 82 (17\%) en « Sciences sociales \& comportementales, psychologie - Géographie humaine $\&$ démographie ». Notre valeur en SHS - Géographie humaine \& démographie est équivalente à celle trouvée par Gorraiz et al., (2016), qui était de $16,8 \%$ en Démographie et Géographie de la Population.

Scopus indexe 12,6\% références de plus que WoS qui couvre 36,8\% (167) des références (Tableau 3). Des 167 références retrouvées par WoS, 132 sont issues des 267 (49\%) références de « Physique, chimie, mathématiques \& sciences de la terre » et 3 des $82(3,6 \%)$ références de « Sciences sociales \& comportementales, psychologie ». Globalement, la différence que nous trouvons entre Scopus et WoS est proche de celles trouvées par Bosman et al., (2006) qui indiquaient une supériorité de $10 \%$ pour Scopus pour les Sciences de la terre ainsi que de la Géographie sociale et de l'Aménagement du territoire. Mais si nous entrons dans le détail de nos chiffres par discipline, les proportions de références retrouvées par les outils fluctuent et nous nous écartons de cette valeur de $10 \%$.

Scopus indexe $16,6 \%$ de références de plus que GeoRef (150). Des 150 références retrouvées par GeoRef 136 sont en « Physique, chimie, mathématiques \& sciences de la terre ». Bosman et al., (2006) avait trouvé $16,3 \%$ en plus pour Scopus par rapport aux titres de périodiques indexés dans GeoRef.

\section{Illustration durecouvremententreles différents outils bibliographiques pour les articles et communications}

Le diagramme de Venn (Figure 1) illustre les taux de recouvrement des outils bibliographiques quant à la recension des 453 articles et communications de l'échantillon.

GS retrouve l'ensemble des 453 références de l'échantillon et le moteur de recherche recouvre à $100 \%$ toutes les autres bases de données. GS recense 183 références « uniques », soit $40 \%$ de l'échantillon. Le corollaire est que $60 \%$ des références sont trouvées par au moins deux des outils bibliographiques.

Parmi les 183 références « uniques » de GS, se trouvent les 68 références qui ont été retrouvées exclusivement par des liens avec ORBi.

97 références $(21,4 \%)$ sont répertoriées par tous les outils bibliographiques. Le pourcentage des références retrouvé par au moins deux des bases de données commerciales est de $38 \%$ ( 174 références). Chacune des bases de données commerciales se distingue des autres bases de données commerciales par un apport supplémentaire de références. Scopus et GeoRef se démarquent respectivement par 55 références $(12,1 \%)$ et 35 références $(7,7 \%)$ tandis que WoS, par seulement 6 références $(1,3 \%)$.

\section{Caractéristiques des références d'articles et communications uniquement répertoriées par GS}

Les 183 références « uniques » de GS se répartissent en deux lots (Tableau 4) : 115 références qui seraient de toute façon retrouvées par GS même si elles 


\section{Légende: \\ Références uniquement couvertes par:

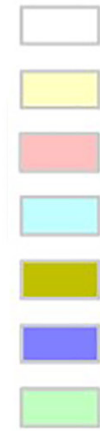 GS \\ GS et Scopus \\ GS et GeoRef \\ GS et WoS \\ GS, WoS et Scopus \\ GS, GeoRef et Scopus \\ GS, GeoRef et WoS}

Références communes à:

GS, Scopus, GeoRef et WoS

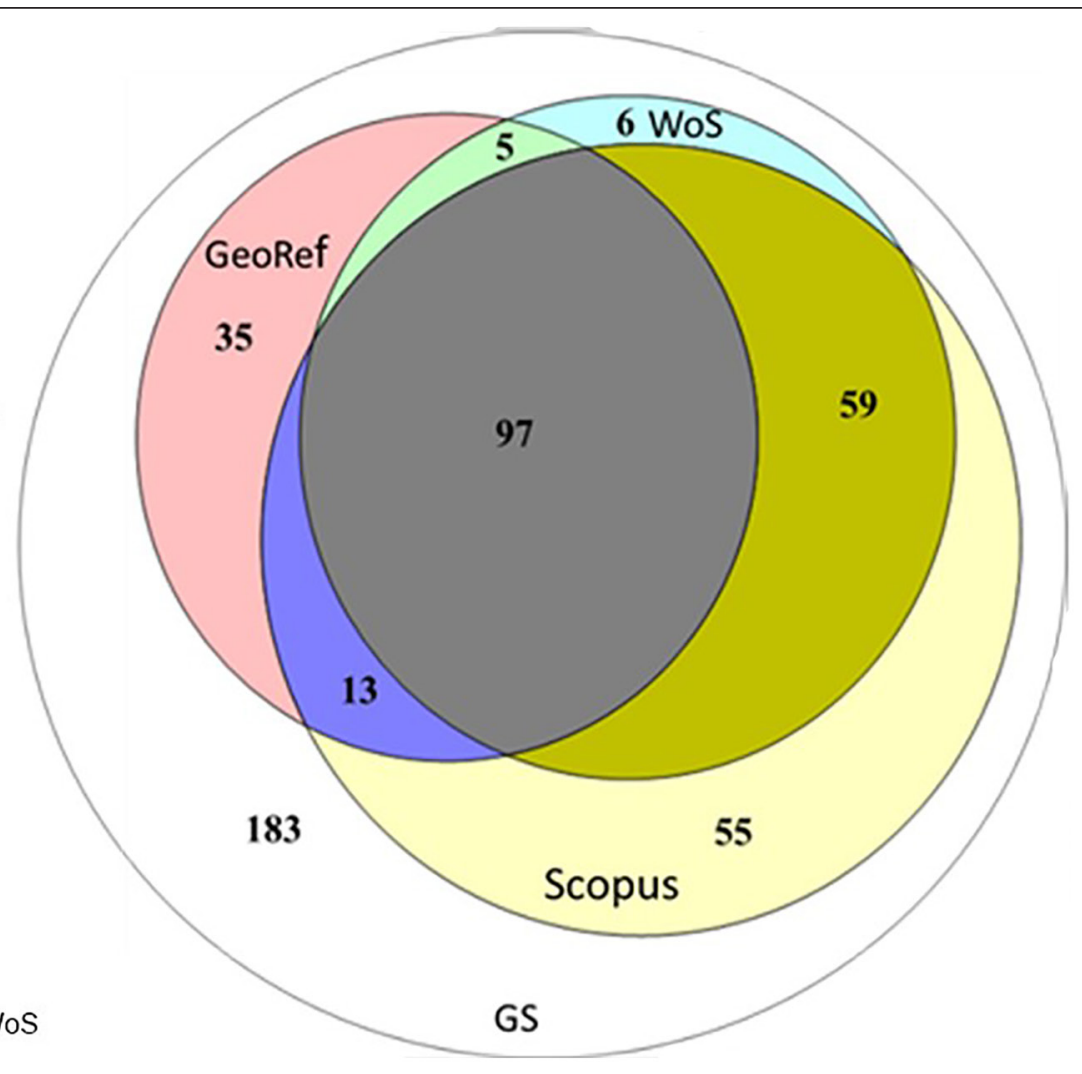

Figure 1. Recouvrement entre les différents outils bibliographiques pour les articles et communications

\begin{tabular}{|l|c|c|c|c|}
\hline & Nb de références uniques & Anglais & Français & Néerlandais \\
\hline GS sans ORBi & 115 & 20 & 94 & 1 \\
\hline GS via ORBi & 68 & 9 & 57 & 1 \\
\hline
\end{tabular}

Tableau 4. Nombre et langue des références « uniques » de GS, sans apport d'ORBi ou via ORBi. Des 115 références « uniques » à GS sans ORBi, 94 sont en langue française (81,7\%), 20 en langue anglaise (17,4 \%) et 1 en portugais.

n'avaient pas été déposées dans ORBi (ci-après : les références de GS sans ORBi) et 68 références qui sont retrouvées par GS uniquement via le moissonnage d'ORBi (ci-après : les références de GS via ORBi).

Notons que 62 des 115 références sont publiées dans le seul Bulletin de la Société Géographique de Liège, périodique peer-reviewed publié en OA, dont la langue de publication est le français et l'anglais. À l'examen du Tableau 4, il apparaît que $\mathbf{6 8}$ références retrouvées par GS via ORBi se répartissent en 57 références $(83,8 \%)$ en français, 9 en anglais $(13,2 \%)$ et 1 en néerlandais (3\%).

Dans ORBi, 46 références de ces 68 références ont été classées dans les « Sciences sociales \& comportementales, psychologie », dont 14 en " Géographie humaine \& démographie » et 4 en « Études régionales \& interrégionales ». Les ST comptent 22 références dont 15 en « Sciences de la terre \& géographie physique $»$.

D'un point de vue qualitatif, il apparait que les 68 références retrouvées par GS via ORBi sont, en grande partie, des publications à caractère scientifique publiées dans des périodiques nationaux, belges et français, en langue française (ex. : Cahiers de l'Urbanisme (Les), Bulletin de la Société Royale Belge d'Études Géologiques et Archéologiques, G.E.O., Géographes Associés, Historiens et Géographes, Mosella, etc.).

\section{DISCUSSION ET CONCLUSIONS}

En référence à la première hypothèse posée dans ce travail, nos résultats montrent que la distribution des types de documents publiés par les membres du Département de Géographie est différente en fonction de l'orientation du sujet des publications vers 
les SHS ou ST. Comme mentionné précédemment, les pratiques de publication spécifiques à la Géographie (Gorraiz et al., 2016) se retrouvent dans notre échantillon également, une majorité de chapitres ou parties de livres en français étant publiés en SHS. En ST les publications majoritaires correspondent aux articles de périodiques et communications en langue anglaise.

La première hypothèse selon laquelle, pour notre échantillon, selon que l'on considère l'orientation vers les SHS ou les ST, la répartition des publications de notre échantillon entre les chapitres ou parties de livres et les articles de périodiques ou communications est nettement différente est confirmée.

Les résultats qui nous ont conduits à la confirmation de notre première hypothèse nous donnent des informations pour tirer les conclusions sur notre deuxième hypothèse : les articles de périodiques sont le type de publication principale quelle que soit la sous-discipline. Sur le total de notre échantillon $75,6 \%$ sont des articles des périodiques et communications publiées dans un périodique. Si l'on fait la distinction entre les SHS et ST, l'article de périodique reste majoritaire : $56,5 \%$ des références en SHS et $86 \%$ en ST.

La deuxième hypothèse selon laquelle, pour notre échantillon, quels que soient la sous-discipline, l'article de périodique est la principale voie de publication est confirmée.

En relation avec notre troisième hypothèse, qui vient compléter les résultats associés aux hypothèses précédentes en ce qui concerne cette fois la langue de publication, nous avons remarqué une dominance des articles de périodiques en anglais en ST (72\%). Moins nombreux dans l'absolu, les articles en SHS sont majoritairement en français (86\%). Nos résultats indiquent aussi que $57 \%$ de chapitres ou parties de livres sont publiés en français tandis que $42 \%$ sont publiés en anglais. Les pratiques de publication du Département de Géographie semblent suivre la tendance générale actuelle en matière de publication scientifique, qui consiste principalement dans la publication d'une majorité d'articles de périodiques en anglais en ST tandis que les publications en SHS les publications sont majoritairement en français qu'elles soient des livres et chapitres de livres ou des articles des périodiques (Castree et al., 2005 ; Johnston et Sidaway, 2015). Cependant, malgré une dominance des articles de périodiques dans les publications des géographes de ULiège, quels que soient l'orientation sous-disciplinaire, les chapitres et parties de livres ainsi que les articles et communications en SHS sont majoritairement en français.

La troisième hypothèse selon laquelle, pour notre échantillon, quels que soient le type de document ou la sous-discipline, l'anglais est la principale langue de publication n'est confirmée que partiellement ;

En relation avec notre quatrième hypothèse nous avons vérifié que GS retrouvait bien $100 \%$ des publications déposées dans ORBi par les géographes de ULiège, ceci grâce au moissonnage du répertoire institutionnel. Sans les références en provenance d'ORBi les résultats de GS serait de 84,8\% de recouvrement pour les articles de périodiques et $33,5 \%$ pour les chapitres ou parties de livres.

Si la production de chapitres ou parties de livres par les géographes de ULiège est environ deux fois plus importante que l'usage que l'on semble généralement faire de ce type de document, c'est grâce au moissonnage d'ORBi que ces références sont toutes visibles et accessibles aux chercheurs via GS.

De manière générale, le moteur de recherche donne une réelle visibilité à ce type de littérature, sans toutefois la couvrir pleinement (voir chapitre $3 \mathrm{de}$ la première partie), tandis que les outils traditionnels répertorient peu, voire très peu, ce type de documents.

La présence de références du Département de Géographie dans ORBi leur donne une plus grande visibilité via GS, le répertoire a un rôle d'autant plus important à jouer dans leur diffusion. C'est en effet grâce à ORBi que cette littérature est visible et accessible aux chercheurs via GS.

La quatrième hypothèse selon laquelle grâce au moissonnage d'ORBi, GS retrouve toutes les publications des géographes de ULiège est confirmée.

En ce qui concerne les références retrouvées uniquement dans GS, exclusivement grâce au mois- 
sonnage d'ORBi, leur nombre est relativement important : pour les chapitres et parties de livres, 96 références $(65,7 \%)$, dont 67 en français, tandis que les articles ou communications comptent 68 références $(15 \%)$, dont 57 en français.

Parmi ces références, les « Sciences sociales \& comportementales, psychologie » sont bien majoritaires pour les chapitres ou de parties de livres avec 74 références, dont la majorité (50) traitent plus précisément de "Géographie humaine \& démographie » ainsi que pour les articles et les communications, 46 références dont 14 en « Géographie humaine \& démographie » et 4 références en «Études régionales \& interrégionales».

La cinquième hypothèse selon laquelle les références retrouvées uniquement dans GS, exclusivement grâce au moissonnage d'ORBi, sont nombreuses, surtout pour la Géographie humaine est confirmée.

$\mathrm{Si}$ on faisait abstraction des références répertoriées grâce à l'indexation d'ORBi uniquement, les performances de GS diminuent de $15 \%$ pour les articles de périodiques et les communications et de près de $70 \%$ pour les chapitres ou parties de livres. Les performances de GS restent tout de même plus élevées que celles des bases de données commerciales.

En prenant nos résultats en considération, nous pouvons constater que GS est un outil bibliographique qui recouvre les références indexées par les bases de données commerciales traditionnelles à $100 \%$. De plus, 183 références, soit $40 \%$ de l'échantillon, sont couvertes par GS uniquement et lui sont « uniques ». GS permet de retrouver des références bibliographiques supplémentaires.

Ces résultats sont comparables aux résultats obtenus sur un autre échantillon de publications en géographie, constitué indépendamment de la production des géographes de ULiège (Ştirbu et al., 2015). Nous avions retrouvé des pourcentages relativement faibles de références " uniques », distinguant WoS de Scopus entre elles, avec un avantage pour Scopus, tandis que GS englobait les résultats des bases de données commerciales. Pour ce qui est de GeoRef, cet outil se démarquait uniquement pour les résultats « uniques » obtenus en Géographie physique.
La sixième hypothèse selon laquelle si on fait abstraction des références en provenance d'OR$B i$, une proportion plus élevée de références est retrouvée par GS en comparaison des bases de données bibliographiques commerciales est confirmée.

Si on regarde le classement des bases de données commerciales, Scopus et WoS suivent GS pour les articles de périodiques et les communications. D'autres études ayant comparé la couverture des bases de données confirment la supériorité de Scopus par rapport à WoS (Bartol et al., 2014 ; Bosman et al., 2006 ; Cavacini, 2015 ; Gavel et Iselid, 2008 ; Vieira et Gomes, 2009). Même si GS recouvre Scopus à $100 \%, 55$ références supplémentaires par rapport à WoS et GeoRef sont à signaler pour Scopus. La couverture des bases de données commerciales dans les deux sous-disciplines principales est plus importante en ST, Scopus se démarque tout de même avec une meilleure couverture en SHS. Nos résultats sont confortés par des études effectuées dans des disciplines différentes (Bartol et al., 2014 ; Bosman et al., 2006 ; Cavacini, 2015).

La septième hypothèse selon laquelle, le recouvrement de WoS et Scopus est très important et seul Scopus se distingue par un apport significatif de références supplémentaires par rapport à WoS, relevant souvent des $S S H$ est confirmée pour les publications des géographes de ULiège.

GeoRef se classe en dernière position pour la couverture des articles de périodiques et des communications. Le classement de GeoRef ne doit cependant pas mener à la conclusion que cet outil bibliographique est à écarter. Même si GS recouvre GeoRef à $100 \%, 35$ références supplémentaires par rapport à WoS et Scopus sont à signaler. $90 \%$ des références retrouvées par GeoRef sont en «Physique, chimie, mathématiques \& sciences de la terre », ce qui indique une bonne couverture en ST. Cette performance rejoint les résultats de notre précédente étude (Ştirbu et al., 2015), où GeoRef occupait la troisième position pour le nombre de références fourni en Géographie physique mais se démarquait par un apport de références « uniques » et de différents types de documents.

La huitième hypothèse selon laquelle, dans l'absolu, GeoRef couvre moins de références que WoS et Scopus mais se distingue par un apport 
de références supplémentaires en Géographie physique par rapport aux deux autres bases de données commerciales est confirmée.

En ce qui concerne la neuvième hypothèse, nos résultats indiquent que $43 \%$ de l'échantillon d'articles de périodiques et communications sont en français. Même si elle n'est pas majoritaire, par rapport aux publications en langue anglaise, la part des références en français est considérable, soit beaucoup plus que l'usage jamais relevé dans la littérature ou accessoirement dans les bibliographies des trois thèses qui ont été étudiées dans la thèse de doctorat de Ştirbu et al., (2015), pourtant réalisées au sein de l'Institution.

À l'analyse des références « uniques » de GS nous avons constaté aussi que le français était prédominant tandis que les périodiques correspondants étaient principalement publiés par la France et la Belgique.

Ce pourcentage important de références en français s'explique par l'affiliation des auteurs à une institution francophone ainsi que par le fait qu'en général, les sujets d'intérêt local et régional sont publiés dans la langue native des auteurs (Gorraiz et al., 2016), dans des périodiques locaux ou nationaux, afin d'atteindre un public local, peut-être autre que les scientifiques et académiques de la discipline. Ainsi, au Bulletin de la Société Géographique de Liège revient 62 des références « uniques » à GS.

Le bon résultat de GS dans le référencement des périodiques francophones locaux est dû, en partie, à la publication en OA de cette littérature ainsi que leur présence dans le répertoire institutionnel ORBi ainsi qu'au moissonnage d'ORBi. La publication de cette littérature en OA contribue également à une plus grande visibilité de ces références.

La neuvième hypothèse selon laquelle du fait de leur présence dans le répertoire institutionnel ORBi, les publications en langue française des géographes liégeois publiées dans les périodiques "locaux» sont plus visibles via GS que via les bases de données commerciales est également confirmée.

En conclusion, considérant notre échantillon de publications des géographes de ULiège, les performances de GS sont toujours supérieures à celles des bases de données. Le répertoire institutionnel ORBi contribue incontestablement à améliorer les performances de GS quant au recouvrement des publications des géographes et à donner une meilleure visibilité de la littérature francophone en géographie dans toute sa diversité. D'une façon plus générale, le référencement des périodiques dans d'autres langues que l'anglais par GS, ainsi que le moissonnage des répertoires institutionnels, pourrait constituer un avantage majeur pour la communauté scientifique en lui fournissant l'accès à un panel de publications plus important, avec une plus grande diversité de sujets et lieux traités, et avec une réduction de l'hégémonie anglaise dans la discipline (Schuermans et al., 2010).

Cependant, en matière d'hégémonie, nous ne pouvons ignorer l'hégémonie de GS justement et des GAFAM ${ }^{6}$ en général. D'où l'importance de développer en parallèle des répertoires institutionnels, des outils de recherche bibliographique indépendants. Un exemple est le moteur de recherche BASE, créé en 2004 dans le cadre du projet Open Archives Initiative par l'Université de Bielefeld (Allemagne) et qui effectue l'indexation automatique des bibliothèques numériques utilisant le protocole Open Archives Initiative Protocol for Metadata Harvesting (OAI-PMH). Un autre exemple, Mosa, mis en œuvre récemment à l'initiative de ULiège Library, vise à mettre en évidence la production scientifique en accès libre des chercheurs des universités et institutions de Belgique et du Luxembourg en interrogeant quotidiennement plus d'une dizaine de répertoires.

\section{NOTES}

${ }^{1}$ Ce travail fait partie d'une thèse doctorale défendue en 2019 (Stirbu, 2019).

${ }^{2}$ Association for Computing Machinery Digital Library: The ACM Digital Library (DL) is the world's most comprehensive database of full-text articles and bibliographic literature covering computing and information technology.

${ }^{3}$ CiteSeerx is an evolving scientific literature digital library and search engine that has focused primarily on the literature in computer and information science. ${ }^{4}$ Base de données spécialisé en Ingénierie développé par Elsevier.

${ }^{5}$ Computer science bibliography : ce service fournit des informations bibliographiques des principaux périodiques et comptes rendus en Informatique. DBLP est géré par Schloss Dagstuhl et fut fondé à l'Université de Trèves en 1993.

${ }^{6}$ Acronyme pour Google Apple Facebook Amazon Microsoft. 


\section{BIBLIOGRAPHIE}

Archambault, É., Côté, G., Struck, B., \& Voorons, M. (2016). Research Impact of Paywalled Versus Open Access Papers. White Papers, Science-Metrix and 1science. http://www.1 science.com/1numbr/

Bartol, T., Budimir, G., Dekleva-Smrekar, D., Pusnik, M., \& Juznic, P. (2014). Assessment of research fields in Scopus and Web of Science in the view of national research evaluation in Slovenia. Scientometrics, 98(2), 1491-1504.

Bosman, J., Mourik, I. van, Rasch, M., Sieverts, E., \& Verhoeff, H. (2006). Scopus reviewed and compared: The coverage and functionality of the citation database Scopus, including comparisons with Web of Science and Google Scholar. Utrecht University Library.

Castree, N., Rogers, A., \& Sherman, D. (2005). Questioning geography: fundamental debates. Wiley-Blackwell.

Cavacini, A. (2015). What is the best database for computer science journal articles? Scientometrics, 102(3), 2059-2071. https://doi.org/10.1007/s11192014-1506-1

Davis, P., \& Fromerth, M. (2007). Does the arXiv lead to higher citations and reduced publisher downloads for mathematics articles? Scientometrics, 71(2), 203-215.

Davis, P. M. (2010). Access, readership, citations: $A$ randomized controlled trial of scientific journal publishing. Cornell University. Retrieved from https://ecommons.cornell.edu/bitstream/handle/1813/17788/Davis\%2C Philip.pdf?sequence=1

Davis, P. M., \& Price, J. S. (2006). eJournal interface can influence usage statistics: implications for libraries, publishers, and Project COUNTER. Journal of the Association for Information Science and Technology, 57(9), 1243-1248.

Davis, P. M., \& Walters, W. H. (2011). The impact of free access to the scientific literature: a review of recent research. Journal of the Medical Library Association: JMLA, 99(3), 208.

De Sutter, B., \& Van Den Oord, A. (2012). To be or not to be cited in computer science. Communications of the ACM, 55(8), 69-75.

Gorraiz, J., Gumpenberger, C., \& Glade, T. (2016). On the bibliometric coordinates of four different research fields in Geography. Scientometrics, 107(2), 873-897. https://doi.org/10.1007/s11192-016-1864-y

Gorraiz, J., Gumpenberger, C., \& Schlögl, C. (2014). Usage versus citation behaviours in four subject areas. Scientometrics, 101(2), 1077-1095. https:// doi.org/10.1007/s11192-014-1271-1

Guerrero-Bote, V. P., \& Moya-Anegón, F. (2014). Relationship between downloads and citations at journal and paper levels, and the influence of language. Scientometrics, 101(2), 1043-1065. https://doi. org/10.1007/s11192-014-1243-5

Harnad, S., Brody, T., Vallières, F., Carr, L., Hitchcock, S., Gingras, Y., Oppenheim, C., Stamerjohanns, H.,
\& Hilf, E. (2004). The access/impact problem and the green and gold roads to open access. Serials Review, 30(4), 310-314.

Jamali, H. R., \& Nabavi, M. (2015). Open access and sources of full-text articles in Google Scholar in different subject fields. Scientometrics, 105(3), 1635-1651.

Johnston, R., \& Sidaway, J. D. (2015). Geography and geographers: Anglo-American human geography since 1945. Routledge.

Meho, L. I., \& Yang, K. (2007). Impact of data sources on citation counts and rankings of LIS faculty: Web of Science versus Scopus and Google Scholar. Journal of the Association for Information Science and Technology, 58(13), 2105-2125.

Mikki, S., Ruwehy, H. A. Al, Gjesdal, Ø. L., \& Zygmuntowska, M. (2018). Filter bubbles in interdisciplinary research: a case study on climate and society. Library Hi Tech, 36(2), 225-236.

Moed, H. F. (2007). The effect of "open access" on citation impact: An analysis of ArXiv's condensed matter section. Journal of the Association for Information Science and Technology, 58(13), 2047-2054.

Norris, M., Oppenheim, C., \& Rowland, F. (2008). Finding open access articles using Google, Google Scholar, OAIster and OpenDOAR. Online Information Review, 32(6), 709-715.

Piwowar, H., Priem, J., Larivière, V., Alperin, J. P., Matthias, L., Norlander, B., Farley, A., West, J. \& Haustein, S. (2018). The state of OA: A large-scale analysis of the prevalence and impact of Open Access articles. PeerJ, 6, e4375. doi:10.7717/ peerj. 4375

Adriaanse, L., \& Rensleigh, C. (2013). Web of Science, Scopus and Google Scholar: A content comprehensiveness comparison. The Electronic Library, 31(6), 727-744.

Schuermans, N., Meeus, B., \& De Maesschalck, F. (2010). Is there a world beyond the Web of Science? Publication practices outside the heartland of academic geography. Area, 42(4), 417-424.

Ştirbu, S. (2019). Développement de l'information électronique et Open Access en Géographie Mise en perspective des performances des outils bibliographiques avec l'usage et la diffusion de la littérature scientifique. Université de Liège, Liège, Belgique.

Ştirbu, S., Thirion, P., Schmitz, S., Haesbroeck, G., \& Greco, N. (2015). The utility of Google Scholar when searching geographical literature: comparison with three commercial bibliographic databases. The Journal of Academic Librarianship, 41(3), 322-329.

Thirion, P. (2016). L'open access, au service de la visibilité de la recherche et des chercheurs? L'expérience d'ORBi. In Open-access à l'université Paris Nanterre: Inauguration du portail HAL.

Vieira, E. S., \& Gomes, J. A. N. F. (2009). A comparison of Scopus and Web of Science for a typical university. Scientometrics, 81(2), 587. 
Coordonnées des auteurs :

Simona STIRBU

ULiège Library, Secteur Sciences \& Techniques Belgique simona.stirbu@uliege.be (contact authors)

Serge SCHMITZ

Département de géographie / Service de géographie rurale (LAPLEC), Sphères

Belgique s.schmitz@uliege.be

Ninfa GRECO

ULiège Library, Secteur Sciences \& Techniques

Belgique n.greco@uliege.be 
